\title{
The Risk of Adverse Events Associated With Atropine Administration During Dobutamine Stress Echocardiography in Cardiac Transplant Patients: A 28-Year Single-Center Experience
}

\author{
JAMES WANG JI, MD², SIQIN YE, MD ${ }^{1}$, JENNIFER HAYTHE, MD ${ }^{1}$, P. CHRISTIAN SCHULZE, \\ MD', and DAICHI SHIMBO, MD1 \\ ${ }^{1}$ Department of Medicine, Columbia University Medical Center, New York, New York \\ ${ }^{2}$ Division of Cardiology, Columbia University Medical Center, New York, New York
}

\begin{abstract}
Background-Although dobutamine stress echocardiography (DSE) is performed in heart transplant patients, the safety profile of atropine administration in DSE in this setting is unclear.

Methods and Results-We identified heart transplant patients who received atropine during DSE from January 1984 to August 2011 at our institution and compared them with a propensityscored matched control group of heart transplant patients who underwent DSE without atropine. Adverse events were defined as significant arrhythmias (sinus arrest, Mobitz type II heart block, complete heart block, ventricular tachycardia, or ventricular fibrillation), hypotension requiring hospitalization, syncope or presyncope, myocardial infarction, and death. Forty-five heart transplant patients (median age 62 years, $82 \%$ male) received $0.2-1 \mathrm{mg}$ atropine during DSE. Of these, 1 patient $(2.2 \%)$ developed temporary complete heart block. No adverse events were identified in the control group of 154 patients who received dobutamine without atropine.
\end{abstract}

Conclusions-Our findings suggest that complete heart block can occur infrequently with the administration of atropine in heart transplant patients undergoing DSE. Therefore, patients should be appropriately monitored for these adverse events during and after DSE.

\section{Keywords}

Atropine; dobutamine; stress echocardiography

\begin{abstract}
Invasive coronary angiography is the recommended primary method for detecting allograft coronary artery disease (CAD) in patients who have undergone orthotopic heart transplantation. ${ }^{1}$ Nevertheless, dobutamine stress echocardiography (DSE) is commonly performed as an alternate modality that can provide complementary assessment of functional perfusion. ${ }^{2-10}$ When dobutamine alone is insufficient to achieve target heart rate during
\end{abstract}

(C) 2013 Elsevier Inc. All rights reserved.

Reprint requests: Daichi Shimbo, MD, Columbia University Medical Center, 622 West 168th Street, PH 9-310, New York, NY 10032. Tel: (212) 342-4490; Fax: (646) 304-7003. ds2231@ columbia.edu.

Disclosures

None. 
stress echocardiography, atropine can be administered to augment the heart rate response. ${ }^{5,7,11}$ However, evidence from small studies suggests that adverse events can occur with the use of atropine in heart transplant patients, including sinus arrest or complete heart block that can result in syncope or require ventricular pacing. ${ }^{12,13}$ Notably, none of those studies were conducted in the setting of concurrent dobutamine infusion. Previous reports of heart transplant patients who received atropine during DSE did not report any significant side effects, but these studies were small and did not primarily examine the safety profile of atropine when given concurrently with dobutamine..$^{5,11,14-17}$ To examine whether atropine can be safely administered during DSE, we examined the use of atropine during DSE for heart transplant patients at our institution.

\section{Methods}

\section{Study Design}

We used the electronic health record (EHR) to retrospectively identify all heart transplant patients who underwent DSE with atropine at the Columbia University Medical Center (CUMC) from January 1, 1984, to August 18, 2011, and selected as control subjects propensity score-matched heart transplant patients who underwent DSE without atropine during the same time period. To accomplish this, we queried DSE reports of all heart transplant patients followed at CUMC during that time period with the use of the terms "dobutamine" and "atropine." From all heart transplant recipients, we identified 2,157 patients who had undergone DSE and of these 45 patients who received both dobutamine and atropine. Of these 45 patients, each underwent exactly 1 DSE during which they received atropine. To identify a suitable control population, we randomly selected a group of 400 transplant patients who underwent DSE but did not receive atropine. Using a standardized data extraction template for both groups (heart transplant patients that received dobutamine and atropine during DSE, and those who received dobutamine only), we manually abstracted from the medical record each patient's demographic and medical covariates at the time of DSE, including age, sex, time since transplant, beta-blocker use, calcium channel blocker use, and history of known transplant CAD (based on left heart catheterization). We performed nearest-neighbor propensity score matching ${ }^{18,19}$ on the 400 randomly selected heart transplant patients who underwent DSE but did not receive atropine to further identify a subset of 154 patients who were matched to those who received both dobutamine and atropine on the aforementioned baseline characteristics (age, sex, time from transplant, beta-blocker use, calcium channel blocker use, and known transplant CAD). For patients in the control group who had undergone >1 DSE, the most recent DSE was included for analysis. The results did not change when earlier DSE studies were used in the analyses for these patients. We then abstracted height, weight, body mass index (BMI), left ventricular ejection fraction (LVEF), history of hypertension, diabetes, or ischemic cardiomyopathy, existing bundle branch block (defined as QRS duration >120 ms) before DSE, and use of immunosuppressants (including corticosteroids, tacrolimus, mycophenolate, sirolimus, and cyclosporine) for all 45 atropine recipients and 154 propensity score-matched patients who did not receive atropine during DSE. 


\section{Dobutamine Stress Echocardiography Protocol}

For DSE, a standard protocol was used for all patients, beginning with dobutamine infusion at $10 \mu \mathrm{g} \mathrm{kg}^{-1} \mathrm{~min}^{-1}$ and increasing by $10 \mu \mathrm{g} \mathrm{kg}^{-1} \mathrm{~min}^{-1}$ every 3 minutes, until a maximum infusion rate of $40 \mu \mathrm{g} \mathrm{kg}^{-1} \mathrm{~min}^{-1}$. For patients who also received atropine, $0.2-1 \mathrm{mg}$ atropine was administered during the final stage. All patients were monitored by electrocardiogram and blood pressure recordings during DSE and monitored on telemetry for $\sim 1$ hour after DSE was completed. We abstracted baseline and peak heart rates and the doses of dobutamine and atropine administered. For adverse outcomes, all patients were monitored for occurrence of adverse events until time of discharge to home, and we manually searched the EHR for any events that occurred within 30 days after DSE for each patient, defined a priori as clinically significant arrhythmias (sinus arrest, Mobitz type II or complete heart block, ventricular tachycardia, or ventricular fibrillation), hypotension requiring hospitalization, syncope or presyncope, myocardial infarction, and death. None of the participants were lost to follow-up during this period.

\section{Statistical Analysis}

Demographic and clinical characteristics were compared for patients who received atropine during DSE and for those who did not. Demographic characteristics that were compared include age, sex, and time since transplant. Clinical characteristics that were compared include BMI, presence of diabetes and hypertension, ischemic cardiomyopathy before transplantation, presence of baseline bundle branch block, LVEF, use of beta-blockers and calcium channel blockers, and presence of transplant CAD based on catheterization. We examined all variables for normality of distribution. In addition, we compared DSE test characteristics, including dobutamine dose, heart rate at rest and peak heart rate, and number of adverse events during DSE between the 2 groups. For normally distributed variables, Pearson chi-square tests or Fisher exact tests were used for comparison between categoric variables, and independent $t$ tests were used for continuous variables. For non-normally distributed variables, nonparametric testing with the use of the Mann-Whitney $U$ test was performed for comparison. A $P$ value of $<.05$ was considered to be statistically significant. All calculations were performed with the use of the statistical packages IBM SPSS Statistics for Windows 17.0 and Stata IC version 10. Propensity score matching was performed using the Stata module psmatch2 by Leuven and Sianesi. ${ }^{20}$

\section{Results}

Of the 45 heart transplant patients who received dobutamine and atropine during DSE, the median (25th-75th percentiles) age at DSE was 62 (49-69) years, and the median (25th75th percentiles) time between heart transplantation and DSE was 7 (4-10) years. Thirtyseven patients (82\%) were male. As expected, the propensity score- matched control group of heart transplant patients who received dobutamine without atropine was not significantly different from the group who received both dobutamine and atropine in age, sex, time since heart transplantation, beta-blocker and calcium channel blocker usage, and known transplant CAD. Other baseline patient characteristics also were similar, including BMI, comorbidities, immunosuppressive medications, prevalence of baseline bundle branch block, and LVEF (Table 1). 
In the group that received dobutamine and atropine, each patient underwent exactly 1 DSE in which they received both medications. These 45 patients all received a maximum rate of dobutamine infusion at $40 \mu \mathrm{g} \mathrm{kg}^{-1} \mathrm{~min}^{-1} ; 24$ (53\%) of the 45 heart transplant patients received a maximum dose of $1 \mathrm{mg}$ atropine. The median (25th-75th percentiles) dose of atropine received was $1(0.5-1) \mathrm{mg}$, corresponding to a weight-based dose of 0.01 (0.006$0.013) \mathrm{mg} / \mathrm{kg}$. In the group that received dobutamine without atropine (Table 2), the median (25th-75th percentiles) maximum rate of dobutamine infusion was $30(20-40) \mu \mathrm{g} \mathrm{kg}-1$ $\min ^{-1}$, which was significantly lower than the dose of dobutamine in patients who did not receive atropine $(P<.005)$. Compared with patients who received both dobutamine and atropine, patients who received dobutamine without atropine had significantly higher resting and peak heart rates $(P<.005$ for both), and were more likely to achieve maximum predicted heart rate for age (MPHR) of $80 \%(P<.005)$ and $85 \%(P<.005$; Fig. 1 ; Table 2$)$.

Regarding adverse events, of the 45 heart transplant patients who received dobutamine and atropine, 1 patient (2.2\%) experienced complete heart block along with ventricular asystole 20 seconds after receiving $0.5 \mathrm{mg}(0.01 \mathrm{mg} / \mathrm{kg})$ atropine, leading to hypotension and syncope (Fig. 2). This patient was a 55-year-old woman 12 years after transplantation with normal allograft cardiac function, baseline right bundle branch block, and no known history of coronary disease, bradycardia, complete heart block, syncope, or hypotension. Chest compressions were administered within 5 seconds of heart block and the patient reverted to normal sinus rhythm with gradual recovery of consciousness. The patient was subsequently hospitalized and an electrophysiology study was conducted the following day which showed no conduction block at or below the level of the His bundle and no evidence of intrinsic conduction disease. She was discharged without further events or need for pacemaker implantation. The remaining 44 patients did not experience any adverse clinical events during or after DSE, and no events were identified within 30 days of DSE. No adverse events were identified in the control group for any of the DSEs performed during the followup period.

\section{Discussion}

The present study represents one of the largest cohort of heart transplant patients who received atropine during DSE and the first to primarily focus on the safety of atropine administration during dobutamine infusion. We examined the use of atropine during DSE in heart transplant patients in a real-world clinical setting, and we found that atropine administration was associated with complete heart block, an infrequent yet idiosyncratic adverse event.

The paradoxic complete heart block induced by atropine alone without dobutamine in heart transplant patients has been described previously. ${ }^{12,13}$ Brunner-la Rocca et al reported that 3 (13\%) of 23 heart transplant patients who received atropine without dobutamine developed syncope with 2nd-degree atrioventricular block or complete heart block 15-150 minutes after receiving atropine. ${ }^{12}$ Similarly, a study by Bernheim et al showed that atropine administration without dobutamine was associated with complete heart block and sinus arrest in 5 (20\%) out of 25 patients in a non-dose-dependent manner, and the lowest atropine dose associated with an adverse event was $0.004 \mathrm{mg} / \mathrm{kg} .{ }^{13}$ Our results extend the 
findings of those earlier studies by examining the risk of complete heart block with atropine in heart transplant patients undergoing concurrent administration of dobutamine.

A few studies of atropine use in heart transplant patients undergoing DSE have been conducted, but they were smaller and did not primarily focus on the risk of adverse events. ${ }^{5,11,14-17}$ A retrospective study by Kociolek et al included 21 transplant patients who received atropine during DSE, and a similar study by Flox et al included 14 transplant patients who also received both agents. ${ }^{11,16}$ The patient populations in those studies were similar to ours, though the study by Flox et al did not include patients using beta-blockers. Although there were slight protocol differences, such as higher doses of atropine and dobutamine in the study by Kociolek et al, no adverse events were reported in either study. In contrast, our study, which primarily focused on the safety of atropine administration in heart transplant patients undergoing DSE, found a possible, but low, risk of paradoxic heart block.

Although several studies have associated atropine with causing bradycardia in nontransplanted patients, ${ }^{21-23}$ reports of atropine associated with high-degree atrioventricular (AV) block are rare. ${ }^{24-26}$ One study described a patient who was administered atropine for anesthesia-induced sinus arrest, ${ }^{26}$ and another case study described a patient that was administered atropine for anesthesia-induced sinus bradycardia ${ }^{24}$; both patients developed high-degree AV block (though not complete heart block) after atropine was given. A third case-series described 12 patients who developed Mobitz type I heart block in the setting of acute inferior wall myocardial infarction; 3 of those patients developed complete heart block after atropine was administered. ${ }^{25}$ In all 3 studies, confounding by indication may have contributed to the results: Atropine was given to patients who had acutely abnormal AV conduction, and therefore the association between atropine and high-degree heart block may merely be a consequence of the deteriorating condition of the patients. In contrast, the present study describes the development of complete heart block after atropine administration in a heart transplant patient receiving dobutamine and whose rhythm was stable.

There is currently no standard recommended duration of monitoring in heart transplant patients once DSE has been completed, particularly for those patients that received atropine. ${ }^{27,28}$ Atropine has a longer half-life (2-3 h) than dobutamine (2 min). ${ }^{22}$ In our study, the one adverse event occurred 20 seconds after receiving atropine. However, given the half-life of atropine, and the idiosyncratic nature of the adverse event, it may be prudent for heart transplant patients who receive atropine to be monitored closely not only during DSE, but also during the period immediately after DSE.

The mechanism of the paradoxic heart block in heart transplant patients who receive atropine is unknown, but earlier investigators speculated that alterations in receptor response to atropine after heart transplantation may be responsible. ${ }^{12,13}$ It has been theorized that changes in muscarinic receptor expression or function may occur after heart transplantation, leading to a parasympathomimetic response after atropine administration. ${ }^{12}$ Alternatively, it has been suggested that atropine could decrease automaticity and prolong the atrial refractory period in poorly re-innervated transplanted hearts. ${ }^{29}$ 
Although our study is focused on safety rather than on efficacy of atropine as a chronotropic agent, we did find that only $2(4.5 \%)$ of 45 patients achieved heart rate $285 \%$ MPHR, which is lower than the findings of earlier studies. ${ }^{11,16}$ This difference could be due in part to protocol differences such as the maximum doses of dobutamine and atropine that were given. Doses of dobutamine and atropine for each patient in our study were determined by the supervising physician. Of the 45 patients, 16 (36\%) were able to achieve $280 \%$ MPHR. As there is some evidence suggesting that this heart rate (80\% MPHR) provides adequate sensitivity for detecting CAD in heart transplant patients, ${ }^{4,17}$ it is possible that physicians at our institution may have targeted a lower maximum heart rate during DSE.

Our study has several possible limitations. Our study is a retrospective analysis of DSE in heart transplant patients performed at a single center, and the dosing of atropine was left at the discretion of the supervising physician for each DSE, which may limit the generalizability of our finding to other institutions. However, the dose of atropine used in our study is similar to or lower than those used in earlier studies, suggesting that the risk of complete heart block may occur even with judicious atropine dosing. It is possible that we did not capture short episodes of complete heart block that occurred hours or more after atropine administration when patients were not monitored. However, we searched for events up to a month after each DSE in our EHR, making it unlikely that any episodes of heart block that we missed would have been clinically significant.

In conclusion, the present study represents one of the largest studies to examine the safety of atropine administration during DSE in heart transplant patients. Our results suggest that in the presence of a chronotropic agent such as dobutamine, complete heart block can occur as an infrequent and idiosyncratic reaction to atropine in heart transplant patients. We recommend that patients who receive atropine should be appropriately monitored after DSE to detect these adverse events. Future studies are needed to better characterize the efficacy of using atropine to augment heart rate response in heart transplant patients undergoing DSE.

\section{Acknowledgments}

Funding: Dr Ye was supported by National Institute of Health (NIH) grant T32HL007854-16 and in part by Columbia University CTSA grant UL1 RR024156 from the National Center for Research Resources/NIH.

\section{References}

1. Costanzo MR, Dipchand A, Starling R, Anderson A, Chan M, Desai S, et al. The International Society of Heart and Lung Transplantation Guidelines for the care of heart transplant recipients. J Heart Lung Transplant. 2010; 29:914-56. [PubMed: 20643330]

2. Smart FW, Ballantyne CM, Cocanougher B, Farmer JA, Sekela ME, Noon GP, et al. Insensitivity of noninvasive tests to detect coronary artery vasculopathy after heart transplant. Am J Cardiol. 1991; 67:243-7. [PubMed: 1990786]

3. Akosah KO, McDaniel S, Hanrahan JS, Mohanty PK. Dobutamine stress echocardiography early after heart transplantation predicts development of allograft coronary artery disease and outcome. $\mathrm{J}$ Am Coll Cardiol. 1998; 31:1607-14. [PubMed: 9626841]

4. Akosah KO, Mohanty PK. Role of dobutamine stress echocardiography in heart transplant patients. Chest. 1998; 113:809-15. [PubMed: 9515861]

5. Bacal F, Moreira L, Souza G, Rodrigues AC, Fiorelli A, Stolf N, et al. Dobutamine stress echocardiography predicts cardiac events or death in asymptomatic patients long-term after heart 
transplantation: 4-year prospective evaluation. J Heart Lung Transplant. 2004; 23:1238-44. [PubMed: 15539121]

6. Spes CH, Klauss V, Mudra H, Schnaack SD, Tammen AR, Rieber J, et al. Diagnostic and prognostic value of serial dobutamine stress echocardiography for noninvasive assessment of cardiac allograft vasculopathy: a comparison with coronary angiography and intravascular ultrasound. Circulation. 1999; 100:509-15. [PubMed: 10430765]

7. McNeill AJ, Fioretti PM, el-Said SM, Salustri A, Forster T, Roelandt JR. Enhanced sensitivity for detection of coronary artery disease by addition of atropine to dobutamine stress echocardiography. Am J Cardiol. 1992; 70:41-6. [PubMed: 1615868]

8. Miller CA, Chowdhary S, Ray SG, Sarma J, Williams SG, Yonan N, et al. Role of noninvasive imaging in the diagnosis of cardiac allograft vasculopathy. Circ Cardiovasc Imaging. 2011; 4:58393. [PubMed: 21934085]

9. Colvin-Adams M, Agnihotri A. Cardiac allograft vasculopathy: current knowledge and future direction. Clin Transplant. 2011; 25:175-84. [PubMed: 21457328]

10. Cai Q, Rangasetty UC, Barbagelata A, Fujise K, Koerner MM. Cardiac allograft vasculopathy: advances in diagnosis. Cardiol Rev. 2011; 19:30-5. [PubMed: 21135600]

11. Flox A, Sanchez V, Delgado JF, Fernandez S, Tello R, Jimenez J, et al. Is atropine infusion necessary to achieve the target heart rate in heart transplant patients during dobutamine stress echocardiography? Transplant Proc. 2002; 34:3241-2. [PubMed: 12493433]

12. Brunner-La Rocca HP, Kiowski W, Bracht C, Weilenmann D, Follath F. Atrioventricular block after administration of atropine in patients following cardiac transplantation. Transplantation. 1997; 63:1838-9. [PubMed: 9210514]

13. Bernheim A, Fatio R, Kiowski W, Weilenmann D, Rickli H, Brunner- la Rocca HP. Atropine often results in complete atrioventricular block or sinus arrest after cardiac transplantation: an unpredictable and dose-independent phenomenon. Transplantation. 2004; 77:1181-5. [PubMed: 15114081]

14. Bacal F, Abuhab A, Mangini S, Fioreli AI, Santos RH, Stolf NG, et al. Dobutamine stress echocardiography in heart transplant recipients' evaluation: the role of reinnervation. Transplant Proc. 2010; 42:539-41. [PubMed: 20304187]

15. Dipchand AI, McCrindle BW, Lee K, West LJ, Smallhorn JF. Dobutamine/atropine stress echocardiography: feasibility, safety and early results in paediatric heart transplant recipients. J Heart Lung Transplant. 2001; 20:232. [PubMed: 11250446]

16. Kociolek LK, Bierig SM, Herrmann SC, Labovitz AJ. Efficacy of atropine as a chronotropic agent in heart transplant patients undergoing dobutamine stress echocardiography. Echocardiography. 2006; 23:383-7. [PubMed: 16686620]

17. Pahl E, Crawford SE, Swenson JM, Duffy CE, Fricker FJ, Backer CL, et al. Dobutamine stress echocardiography: experience in pediatric heart transplant recipients. J Heart Lung Transplant. 1999; 18:725-32. [PubMed: 10452350]

18. Austin PC. An introduction to propensity score methods for reducing the effects of confounding in observational studies. Multivar Behav Res. 2011; 46:399-424.

19. Rosenbaum PR, Rubin DB. Constructing a control group using multivariate matched sampling methods that incorporate the propensity score. Am Stat. 1985; 39:33-8.

20. Leuven, E.; Sianesi, B. Statistical Software Components S432001. Boston College Department of Economics; 2003. Psmatch2: Stata module to perform full Mahalanobis and propensity score matching, common support graphing, and covariate imbalance testing. revised 19 Jul 2012

21. American Heart Association. 2005 American Heart Association (AHA) guidelines for cardiopulmonary resuscitation (CPR) and emergency cardiovascular care (ECC) of pediatric and neonatal patients: pediatric advanced life support. Pediatrics. 2006; 117:e1005-28. [PubMed: 16651281]

22. Atropine. Lexi-Drugs Online [Internet Database]. Hudson, OH: Lexi-Comp, Inc; 1978-2013. Available from: http://online.lexi.com/crlsql/servlet/crlonline [Accessed September 1, 2013]

23. Das G, Talmers FN, Weissler AM. New observations on the effects of atropine on the sinoatrial and atrioventricular nodes in man. Am J Cardiol. 1975; 36:281-5. [PubMed: 1101675] 
24. Chin KJ, Seow SC. Atrioventricular conduction block induced by low-dose atropine. Anaesthesia. 2005; 60:935-6. [PubMed: 16115264]

25. Lewin RF, Kusniec J, Sclarovsky S, Strasberg B, Arditti A, Pinchas A, et al. Alternating Wenckebach periods in acute inferior myocardial infarction: clinical, electrocardiographic, and therapeutic characterization. Pacing Clin Electrophysiol. 1986; 9:468-75. [PubMed: 2426663]

26. Maruyama K, Mochizuki N, Hara K. High-degree atrioventricular block after the administration of atropine for sinus arrest during anesthesia. Can J Anaesth. 2003; 50:528-9. [PubMed: 12734175]

27. Becher H, Chambers J, Fox K, Jones R, Leech GJ, Masani N, et al. BSE procedure guidelines for the clinical application of stress echo-cardiography, recommendations for performance and interpretation of stress echocardiography: a report of the British Society of Echocardiography Policy Committee. Heart. 2004; 90(Suppl 6):23-30. vi.

28. Geleijnse ML, Krenning BJ, Nemes A, van Dalen BM, Soliman OI, Ten Cate FJ, et al. Incidence, pathophysiology, and treatment of complications during dobutamine-atropine stress echocardiography. Circulation. 2010; 121:1756-67. [PubMed: 20404267]

29. Epstein AE, Hirschowitz BI, Kirklin JK, Kirk KA, Kay GN, Plumb VJ. Evidence for a central site of action to explain the negative chronotropic effect of atropine: studies on the human transplanted heart. J Am Coll Cardiol. 1990; 15:1610-7. [PubMed: 2345242] 


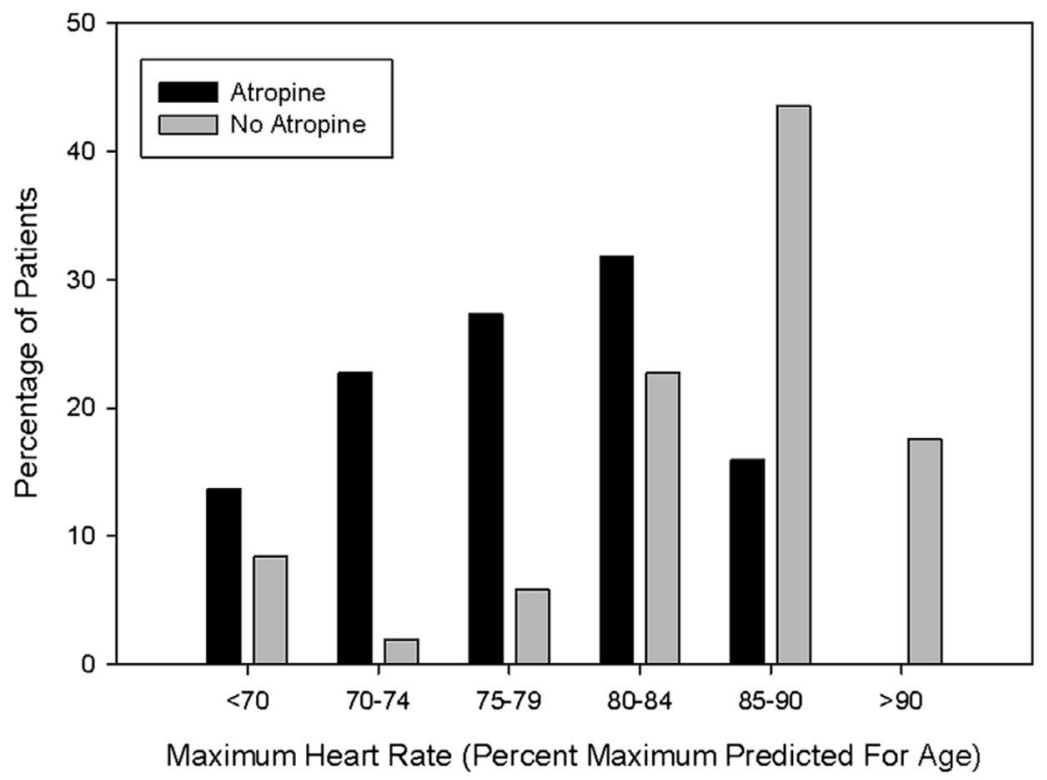

Fig. 1.

Distribution of maximum heart rates expressed as maximum predicted heart rate for age in heart transplant patients who did and did not receive atropine during dobutamine stress echocardiography. Percentage of patients represents the percentage within each group of patients. 


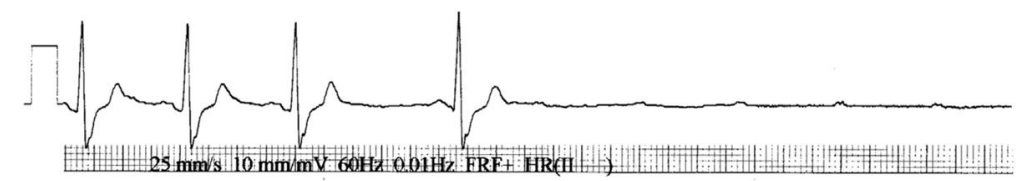

Fig. 2.

Rhythm strip (lead II) of a heart transplant patient with complete heart block with ventricular asystole, recorded 20 seconds after atropine was administered during dobutamine stress echocardiography. 


\section{Table 1}

\section{Patient Characteristics}

\begin{tabular}{|c|c|c|c|}
\hline & $\begin{array}{c}\text { Patients Who Did Not } \\
\text { Receive Atropine }(\mathrm{n}=\mathbf{1 5 4})\end{array}$ & $\begin{array}{c}\text { Patients Who Did } \\
\text { Receive Atropine }(n= \\
\text { 45) }\end{array}$ & $P$ Value* \\
\hline Median age, y (25-75th percentiles) & $61(51.5-70.5)$ & $62(49-69)$ & .55 \\
\hline Median time since transplant at time of DSE, y (25-75th percentiles) & $7(3.5-12)$ & $7(4-10)$ & .69 \\
\hline Male gender, $\mathrm{n}(\%)$ & $134(87 \%)$ & $37(82 \%)$ & .62 \\
\hline Mean BMI, $\mathrm{kg} / \mathrm{m}^{2}$ (SD) & $27.7(4.9)$ & $28.0(5.4)$ & .73 \\
\hline Diabetes, $\mathrm{n}(\%)$ & $81(53 \%)$ & $22(49 \%)$ & .66 \\
\hline Hypertension, $\mathrm{n}(\%)$ & $67(44 \%)$ & $20(44 \%)$ & .91 \\
\hline Ischemic cardiomyopathy before heart transplantation, $\mathrm{n}(\%)$ & $50(33 \%)$ & $16(39 \%)$ & .30 \\
\hline On beta-blocker, $\mathrm{n}(\%)$ & $49(32 \%)$ & $15(36 \%)$ & .85 \\
\hline On calcium channel blocker, $\mathrm{n}(\%)$ & $20(13 \%)$ & $4(21 \%)$ & .46 \\
\hline Baseline bundle branch block, $\mathrm{n}(\%)$ & $33(21 \%)$ & $7(16 \%)$ & .39 \\
\hline Mean no. of immunosuppressants (SD) & $2.7(0.5)$ & $2.7(0.5)$ & .47 \\
\hline Mean left ventricular ejection fraction, $\%$ (SD) & $56(8)$ & $56(7)$ & .94 \\
\hline Has transplant $\mathrm{CAD}$ by catheterization, $\mathrm{n}(\%)$ & $9(5.8 \%)$ & $2(4.4 \%)$ & .72 \\
\hline
\end{tabular}

BMI, body mass index; CAD, coronary artery disease, DSE, dobutamine stress echocardiography.

${ }^{*} P$ values were calculated with the use of chi-square tests for categoric variables and Student $t$ tests for continuous variables, except for age and time since transplantation, for which Mann-Whitney $U$ tests were used owing to skewed distributions. 
Table 2

Test Characteristics in Heart Transplant Patients Who Did and Did Not Receive Atropine During DSE

\begin{tabular}{|c|c|c|c|}
\hline & $\begin{array}{l}\text { Patients Who Did Not } \\
\text { Receive Atropine }(n=154)\end{array}$ & $\begin{array}{l}\text { Patients Who Did Receive } \\
\text { Atropine }(n=45)\end{array}$ & $P$ Value* \\
\hline $\begin{array}{l}\text { Median maximum dobutamine dose, } \mu \mathrm{g} \mathrm{kg}^{-1} \mathrm{~min}^{-1}(25-75 \text { th } \\
\text { percentiles) }\end{array}$ & $30(20-40)$ & $40^{\dagger}$ & $<.005$ \\
\hline Mean heart rate at rest, bpm (SD) & $86(12)$ & $76(9)$ & $<.005$ \\
\hline Mean peak heart rate, bpm (SD) & $135(17)$ & $123(14)$ & $<.005$ \\
\hline Mean peak heart rate, $\%$ MPHR (SD) & $85(9)$ & $76(7)$ & $<.005$ \\
\hline Achieved 80\% MPHR, n (\%) & $128(83 \%)$ & $16(36 \%)$ & $<.005$ \\
\hline Achieved 85\% MPHR, n (\%) & $97(63 \%)$ & $2(4.5 \%)$ & $<.005$ \\
\hline
\end{tabular}

bpm, beats per minute; MPHR, maximum predicted heart rate for age.

${ }^{*} P$ values were calculated with the use of chi-squared tests for categoric variables and Student $t$ tests for continuous variables, except for dobutamine dose, for which Mann-Whitney $U$ tests were used owing to skewed distributions.

${ }^{\dagger}$ All patients in this group received $40 \mu \mathrm{g} \mathrm{kg}-1 \mathrm{~min}^{-1}$. 